\title{
Anti-inflammatory effects of Metformin improve the neuropathic pain and locomotor activity in spinal cord injured rats: introduction of an alternative therapy
}

\author{
Khashayar Afshari ${ }^{1,2,3} \cdot$ Amir Dehdashtian ${ }^{1,2} \cdot$ Nazgol-Sadat Haddadi $^{1,2} \cdot$ Arvin Haj-Mirzaian $^{2,4} \cdot$ Arad Iranmehr $^{2,5}$. \\ Mohammad Ali Ebrahimi ${ }^{2,6}$. Seyed Mohammad Tavangar ${ }^{7,8} \cdot$ Hedyeh Faghir-Ghanesefat $^{2} \cdot$ \\ Fatemeh Mohammadi ${ }^{2,3} \cdot$ Nastaran Rahimi ${ }^{2} \cdot$ Abbas Norouzi Javidan $^{3,9} \cdot$ Ahmad Reza Dehpour $^{2,10}$
}

Received: 12 January 2018 / Revised: 28 May 2018 / Accepted: 29 May 2018 / Published online: 29 June 2018

(c) International Spinal Cord Society 2018

\begin{abstract}
Study design This is an animal study.

Objectives Metformin is a safe drug for controlling blood sugar in diabetes. It has been shown that metformin improves locomotor recovery after spinal cord injury (SCI). Neuropathic pain is also a disturbing component of SCI. It is indicated that metformin has neuroprotective and anti-inflammatory effects, which attenuate neuropathic pain and hyperalgesia in injured nerves. Thus, we evaluated metformin's therapeutic effects on SCI neuroinflammation and its sensory and locomotor complications. Meanwhile, results were compared to minocycline, an anti-neuroinflammation therapy in SCI.

Setting Experimental Medicine Research Center, Tehran University of Medical Sciences, Iran

Methods In an animal model of SCI, 48 male rats were subjected to T9 vertebra laminectomy. Animals were divided into a SHAM-operated group and five treatment groups. The treatments included normal saline as a vehicle control group, minocycline $90 \mathrm{mg} / \mathrm{kg}$ and metformin at the doses of 10,50 and $100 \mathrm{mg} / \mathrm{kg}$. Locomotor scaling, behavioral tests for neuropathic pain and weight changes were evaluated and compared through a 28-days period. At the end of the study, tissue samples were taken to assess neuroinflammatory changes.

Results Metformin $50 \mathrm{mg} / \mathrm{kg}$ improved the locomotors ability $(p<0.001)$ and decreased sensitivity to mechanical and thermal allodynia $(p<0.01)$. These results were compatible with minocycline effect on SCI $(p>0.05)$. While metformin led to weight loss, both metformin and minocycline significantly decreased neuroinflammation in the assessment of cord tissue histopathology, and levels of TNF- $\alpha$ and interleukin- $1 \beta(p<0.001)$.

Conclusions Metformin could be considered as an alternative therapeutic agent for SCI, as it potentially attenuates neuroinflammation, sensory and locomotor complications of cord injury.
\end{abstract}

Electronic supplementary material The online version of this article (https://doi.org/10.1038/s41393-018-0168-x) contains supplementary material, which is available to authorized users.

Ahmad Reza Dehpour

dehpoura@sina.tums.ac.ir

dehpour@yahoo.com

1 MD-MPH, Tehran University of Medical Sciences, Tehran, Iran

2 Experimental Medicine Research Center, Tehran University of Medical Sciences, Tehran, Iran

3 Brain and Spinal Cord Injury Research Center, Neuroscience Institute, Tehran University of Medical Sciences, Tehran, Iran

4 MD, Shahid Beheshti University of Medical Sciences, Tehran, Iran

5 Neurosurgery Resident, School of Medicine, Tehran University of
Medical Sciences, Tehran, Iran

6 Phar.D., Tehran University of Medical Sciences, Tehran, Iran

7 Chronic Diseases Research Center, Endocrinology and Metabolism Population Sciences Institute, Tehran University of Medical Sciences, Tehran, Iran

8 Professor of Pathology, Department of Pathology, Dr. Shariati Hospital, Tehran University of Medical Sciences, Tehran, Iran

9 Associate professor of Physiology, Ph.D. in Physiology, Tehran University of Medical Sciences, Tehran, Iran

10 Professor of Pharmacology, Ph.D. in Pharmacology, Tehran University of Medical Sciences, Tehran, Iran 


\section{Introduction}

There are two defined pathological pathways that lead into neuronal damages in SCI: the primary mechanism is the mechanical cord injury happening due to trauma to the spinal cord; the secondary damage occurs due to edema, inflammation, excitotoxicity, autophagy disruption and neuronal apoptosis. Secondary damage plays a critical role in the morbidities of SCI [1]. Different mediators such as TNF- $\alpha$, interleukin- $1 \beta$, and nitric oxide are involved in the mechanisms of secondary damage. It has been suggested that after the traumatic injury mediators such as nitric oxide trigger immune responses which lead to the activation of microglia with consequent release of proinflammatory factors including TNF- $\alpha$ and interleukin$1 \beta[1,2]$. These cytokines cause increased inflammation, neuronal apoptosis and immune cell migration to the site of injury, which promotes prolonged SCI neuroinflammation. This neuroinflammation can lead to progressive neurodegeneration, and functional impairments such as neuropathic pain [1,2]. Thus, indeed blocking the molecular pathways leading to inflammation could be of benefit to improve functional recovery in SCI patients.

Neuropathic pain is an important component in SCI affecting about $80 \%$ of the patients [3]. Significantly decreasing quality of life, this pain could lead to prominent disability and delay in rehabilitation. These patients experience a variety of neuropathic pain conditions such as hyperalgesia [3]. Although there are no definitive treatments for SCI neuropathic pain, recent investigation has shown significant attenuation of this pain through the administration of minocycline, a potent drug that has been previously shown to have significant neuroprotective effects via TNF- $\alpha$, interleukin-1 $\beta$ (IL-1 $\beta$ ) and microglial inhibition. In addition, mammalian target of rapamycin (mTOR) inhibitors have similar potency in SCI treatment [3-5].

Metformin inhibits mTOR and is a well-known drug for controlling diabetes type 2 [6]. Recently, Di Zhang et al. and C.Wang et.al. showed that metformin regulates the process of autophagy and locomotor recovery after spinal cord injury through AMP-activated protein kinase (AMPK) activation and mTOR inhibition [7, 8]. Likewise, metformin has neuroprotective and anti-inflammatory effects in the central and peripheral nervous systems [9-11]. It has also been shown that metformin attenuates neuropathic pain and hyperalgesia in the spared nerve injury model via decreased activation of microglia [12]. Considering the potential efficacy of minocycline and its possible common therapeutic pathways with metformin, we decided to compare the metformin's efficacy on SCI complications with minocycline.

\section{Methods}

\section{Animals}

This study was performed on 48 male Sprague-Dawley rats weighing $240-260 \mathrm{~g}$ provided from Pasteur Institute (Tehran, Iran). Every two Rodents were kept in separate housing cages with a room temperature $23 \pm 2{ }^{\circ} \mathrm{C}$ and $50 \pm 5 \%$ humidity in a $12 \mathrm{~h}$ day/night cycle; they had free access to tap water and rat chow pellets. Animal weight changes and occurrence of complications were also recorded in this study.

\section{Drugs}

Metformin, minocycline, cefazolin, buprenorphine, and xylazine were purchased from Sigma (St. Louis, Missouri, United States). Ketamine $\mathrm{HCl}$ was purchased from Gedeon Richter Ltd (Budapest, Hungary). Drugs were dissolved in physiologic saline and administered intraperitoneally at the time of surgery, with a volume of $1 \mathrm{ml} / \mathrm{kg}$.

\section{Induction of SCI}

Anesthesia was induced by a mixture of ketamine $(80 \mathrm{mg} / \mathrm{kg})$ and xylazine $(10 \mathrm{mg} / \mathrm{kg})$. Animals were kept on a sterile heating pad during the time of surgery. Secured in a prone position rodents were subjected to $\mathrm{T} 9$ vertebra complete laminectomy followed by a spinal cord contusion injury induced by $60 \mathrm{~s}$ compression of the cord by an aneurysmal clip (YASARGIL ${ }^{\circledR}$ Aneurysm clip system, Titanium mini clips FT712T; closing force, $110 \mathrm{~g}$ [1.08 N]; $4.7 \mathrm{~mm}$ Blade length; $3.8 \mathrm{~mm}$ maximum opening diameter). Afterward, the wounds were sutured and animals received cefazolin $(20 \mathrm{mg} /$ $\mathrm{kg})$ and buprenorphine $(0.1 \mathrm{mg} / \mathrm{kg})$ and were kept in a $35^{\circ} \mathrm{C}$ incubator until regaining consciousness. Post-operative care was provided by administration of saline $(2 \mathrm{ml})$, cefazolin $(20 \mathrm{mg} / \mathrm{kg})$ and buprenorphine $(0.1 \mathrm{mg} / \mathrm{kg})$ from day 0 to 6 , and twice a day manual voiding of the bladder until redeeming the function.

\section{Study groups}

Animals were divided to six groups, a control group receiving only vehicle, i.e., saline $(0.9 \%)$, metformin treatment groups, with intraperitoneal drug administration of different single doses of 10,50 and $100(\mathrm{mg} / \mathrm{kg})$ at the time of injury, for determination of the most efficient dose response, minocycline group treated at a dose of $90 \mathrm{mg} / \mathrm{kg}$ immediately after SCI and two doses of $45 \mathrm{mg} / \mathrm{kg}$ every $12 \mathrm{~h}$ [13], and finally a SHAM-operated group, which having no contusion injury received vehicle and other postoperative treatments. All of the data in this manuscript were 
gathered by different investigators blinded to the treatment groups.

\section{Locomotor rating}

The hind limb locomotor activity of the rats was scored on day 0 before the surgery, and 1, 3, 5, 7, 14, 21 and 28 days after SCI based on Basso, Beattie, Bresnahan (BBB) Locomotor Rating Scale. The scorings ranged from 0 to 21 based on weight bearing, limb movements, coordination, joint movements, coordination and operation of individual joints ( 0 indicates for no motor function, while 21 stands for full motor ability). Two investigators, blinded to the treatments, observed and scored each rat placed in an open field for 10 min. Scorings were given for every hind limb, and the mean score was documented for each rat at each session [14].

\section{Neuropathic pain assessment}

\section{Thermal allodynia}

Tail-flick latency (TFL) was measured using a Tail-Flick Analgesia Meter (IITC life science model 33t; Los Angeles, California, USA) at day 0 before the surgery and 7, 14, 21, and 28 days post-operation. After an acclimatization time of 45-min, TFL was evaluated by exposing the dorsum of the rodent's tail to a beaming heat source; while conscious, the time taken for each animal to move its tail from the painful thermal incitement was recorded. In this regard for appropriate baseline intensity, each animal was subjected to five tests. The strength of the incitement was adjusted so that TFL would be between 7 and $8 \mathrm{~s}$ in unharmed animals, thus a cutoff time of $8 \mathrm{~s}$ was set to prevent injury. The mean withdrawal time was then calculated and documented by following tail-flick tests in each rodent.

\section{Mechanical allodynia}

The mechanical hyperalgesia in the plantar hind paws was evaluated by using von Frey filaments (Bioseb, USA). In this method, the cutaneous sensitivity to different filament stimulation for both hind paws was recorded as $50 \%$ withdrawal threshold in each rat and then the paws average score, as grams, was documented as a single value, at day 0 before the surgery and $7,14,21$, and 28 days after SCI.

\section{Histopathological scoring}

After 28 days of assessments, all animals were anesthetized with Ketamine $(100 \mathrm{mg} / \mathrm{kg})$ and xylazine $(10 \mathrm{mg} / \mathrm{kg})$. Then the thorax was incised and with the heart exposed $150 \mathrm{ml}$ of phosphate buffer saline (PBS) $(\mathrm{pH}=7.4)$ was transcardially perfused after incising the right atrium was to permit exsanguination. The perfusion was followed by $250 \mathrm{ml}$ of $4 \%$ paraformaldehyde (PFA) in half of the rodents in each group. While in the other half, the spinal cord was dissected, frozen in liquid nitrogen and held in a $-70{ }^{\circ} \mathrm{C}$ fridge for tissue cytokine assessment. The dissected spinal cord in the first half though was post-fixed in $4 \%$ paraformaldehyde for $72 \mathrm{~h}$ in PFA solution.

Spinal cord sections provided from these samples were further stained with hematoxylin and eosin (H\&E) to study pathological changes. Three sections delivered from each rodent were primarily deparaffinized at $70{ }^{\circ} \mathrm{C}$ for $20 \mathrm{~min}$ and xylene solution for $3 \mathrm{~min}$, then rehydrated $(2 \mathrm{~min})$ and rinsed in tap water. Slides were rinsed in hematoxylin solution (Sigma, Chemical Co) for $15 \mathrm{~min}$ to stain nuclei then rinsed in tap water. 2 min eosin staining was performed followed by rinsing in 90, 96 and $100 \%$ ethanol for $2 \mathrm{~min}$. Afterward, samples were immersed in xylene and mounting medium (Merck, Germany). Finally, slides were evaluated by a blinded, expert pathologist for quantitative analysis and histopathological scoring of the spinal cord impairment based on four factors including immune cells infiltration and inflammation, hemorrhage, axonal vacuolation and cyst formation. For histopathological scoring, we generated a calibrated transparent grid with a Zeiss Axiovision image analysis software (Carl Zeiss, Oberkochen, Germany) $5+$ $100 / 100 \mathrm{~mm}$ scale over tissue samples on $\times 100$ magnification. Each gridline represented $0.1 \mathrm{~mm}$ displayed on the screen. A gridline was centered on the injury site and the area of $2 \mathrm{~mm}$ rostral and $2 \mathrm{~mm}$ caudal to this point was selected for analysis. The analysis was average measure on three different areas of a sample $100 \mu \mathrm{m}$ apart. Histopathologic assessment scored from 0 to 3 for the presence of vacuolization, cyst formation, hemorrhage, and inflammation. Where 0 was indicative of no evidence, 1 was mild, 2 was moderate, and 3 was severe. Scores then presented as a cumulative score of $0-12[15,16]$.

\section{Assessment of inflammatory mediators}

For measuring the inflammatory mediators level in the spinal cord specimens, the samples were dissected out and homogenized in lysis buffer; the homogenized samples were centrifuged at 4000 r.p.m. for $15 \mathrm{~min}$ at $4{ }^{\circ} \mathrm{C}$. Enzyme-linked immunosorbent assay (ELISA; Abcam, Cambridge, UK) were then used to determine the levels of TNF- $\alpha$ and IL-1 $\beta$.

\section{Statistical analysis}

The sample size was calculated using $G^{*}$ power software version 3 . In this regard, considering the power of the study reaching 0.8 and $\alpha=0.05$, a sample size of $6-8$ animals for each group was calculated to be adequate for the current study. Differences in locomotor scores and 

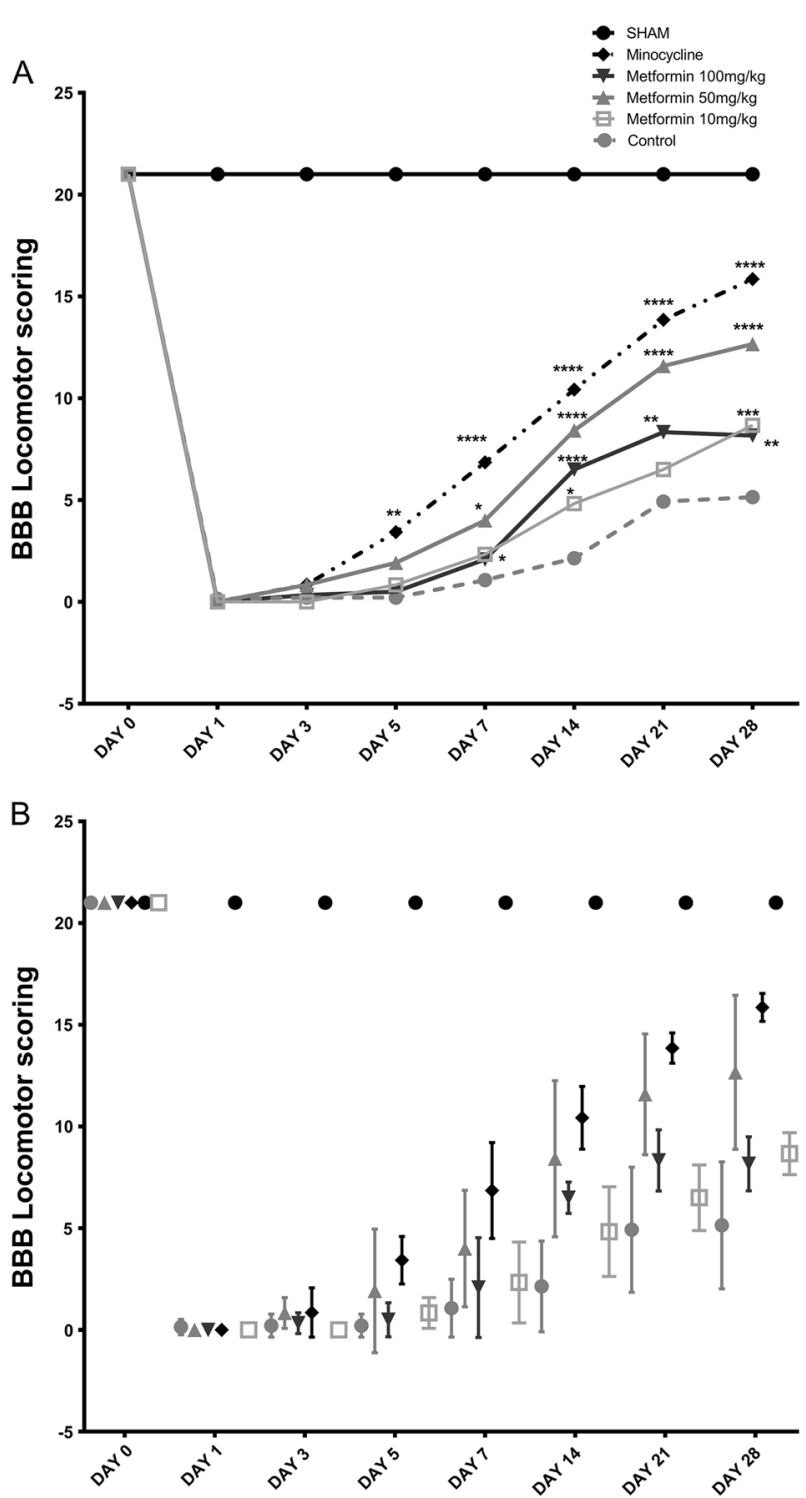

Fig. 1 Line chart of BBB locomotor scores of rats treated with metformin $(10,50$ and $100 \mathrm{mg} / \mathrm{kg})$ and minocycline. a Metformin at the dose of $50 \mathrm{mg} / \mathrm{kg}$ improved hind limb locomotor recovery through 28 days. There was no significant difference between metformin $(50 \mathrm{mg} / \mathrm{kg})$ and minocycline treatments over 28 days of observation. b The data are presented as mean $\pm \mathrm{SD}$ (6-8 rats per groups). $* P<$ $0.05, * * P<0.01, * * * P<0.001$, and $* * * * P<0.0001$ compared to the control group on the same day

neuropathic pain assessments throughout the evaluation time period were analyzed by repeated measures analysis of variance (ANOVA) followed by Tukey's post hoc test. Differences in pathologic scorings and tissue cytokine levels were analyzed by analysis of variance (ANOVA) test followed by Tukey's post hoc test. Statistical Analyses were performed by SPSS version 24 (Chicago, Illinois, United States) and $p$ value $<0.05$ was considered to be statistically significant. The correlation of locomotor score and pain assessments was also analyzed in order to evaluate the effect of locomotion changes on pain response.

\section{Statement of ethics}

We certify that all applicable institutional and governmental regulations concerning the ethical use of animals were followed during the course of this research. All animal studies were handled according to the Declaration of Helsinki principles and followed by the International Association for the Study of Pain guidelines for Animal Experiments. This study has ethical approval by Tehran University of Medical Sciences ethics committee (IRB code: 8911215014282528).

\section{Results}

\section{Locomotor scaling}

One day after the surgery, the mean locomotor scores significantly dropped from (Mean: 21, SD: 0) to (Mean: 0.031, SD: 0.17) in all treatment groups $(p<0.001)$ except for the SHAM-operated animals. A two-way repeated measure ANOVA was conducted that examined the effect of treatment group and time (28 days) on BBB locomotor scale. While time was shown to be independently effective in score changes $(p<0.001)$, there was a statistically significant interaction between the treatment group and time on BBB locomotor scale $(p<0.001)$. Results showed that there was a significant difference in locomotor scale between different treatment groups $(p<0.001)$. Multiple comparisons using Tukey post hoc test indicated that the mean locomotor scores compared to control group was improved in metformin $50 \mathrm{mg} / \mathrm{kg}$ (Mean difference: 3.195, S.E: $0.553)(p<0.001)$ and minocycline (Mean difference: 4.67, S.E 0.531) $(p<0.001)$, while there was no significant difference between these treatments $(p=0.107)$. Although, none of the effective interventions mean locomotor scaling were similar to the SHAM-operated group $(p<0.001)$. (Fig. 1).

\section{Neuropathic pain}

\section{Tail-flick latency}

A week after the surgery (day 7), assessment of the SCI groups for response to thermal incitements (tail-flick test) revealed a significant decline in the mean TFL (Mean difference: 1.13 , SD difference: $0.91, p<0.001$ ). Evaluation of weekly changes in latency with repeated measure ANOVA showed that while time $(p<0.001)$ and its interaction with treatment group $(p<0.001)$ were significantly effective in 

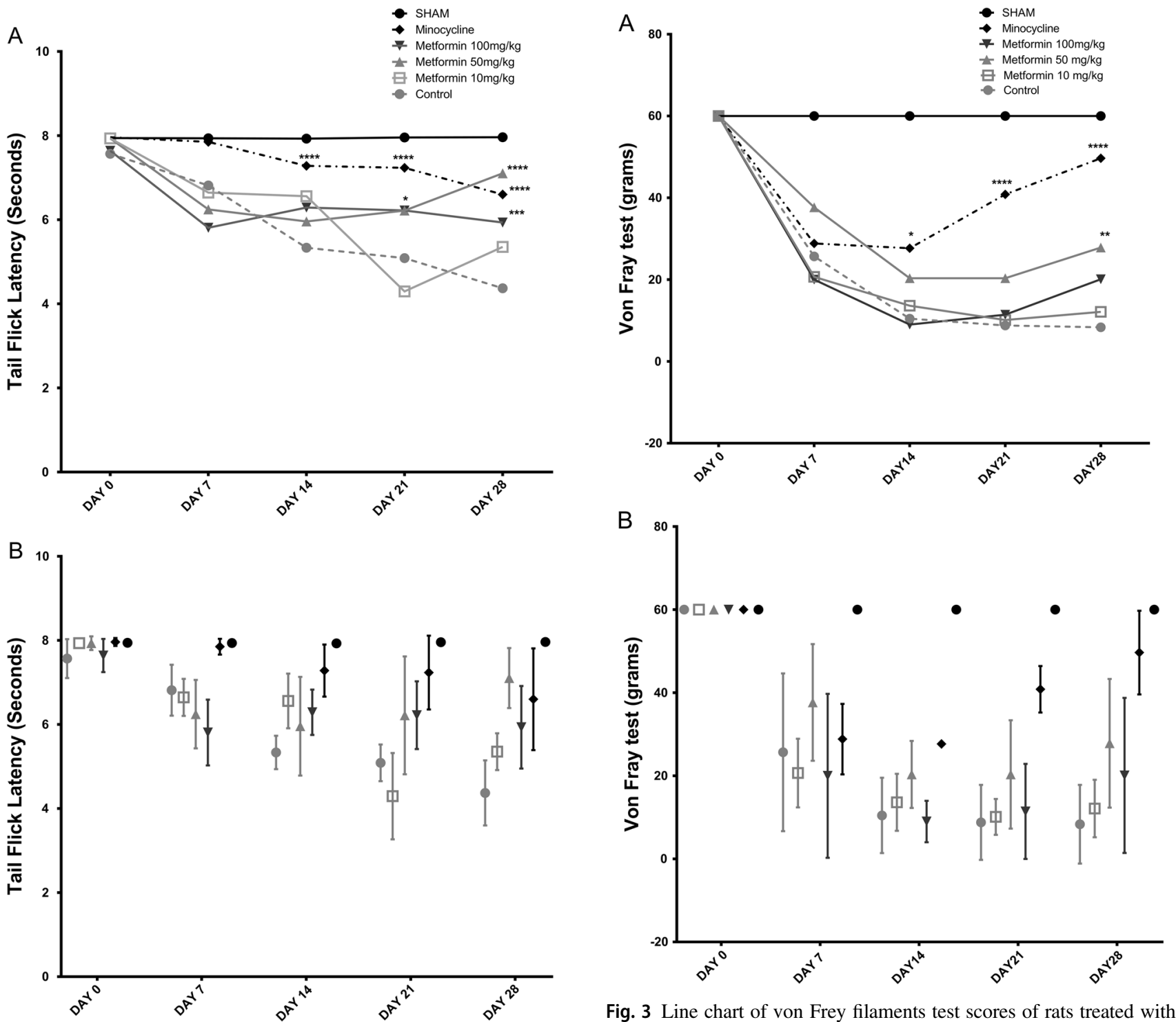

Fig. 2 Line chart of the mean tail flick latencies of rats treated with metformin $(10,50$ and $100 \mathrm{mg} / \mathrm{kg})$ and minocycline. a There was a significant improvement in TFL scoring in metformin $(50 \mathrm{mg} / \mathrm{kg}$ ) group through 28 days after surgery. There was no significant difference between metformin $(50 \mathrm{mg} / \mathrm{kg})$ and minocycline-treated animals in the study period. $\mathbf{b}$ The data are presented as mean \pm SD $(6-8$ rats per groups). $* P<0.05$, $* * * P<0.001$, and $* * * * P<0.0001$ compared to the control group on the same day

TFL changes, there was a significant difference between the efficacy of different drugs $(\mathrm{F}(5,31)=25.689, p<0.001)$. Metformin $50 \mathrm{mg} / \mathrm{kg}$ and minocycline were similarly $(p=$ $0.052)$ effective $(p<0.001)$ on increasing the latency and inhibiting the nociceptive phenomena induced by SCI over a 4-weeks time period (Fig. 2).

\section{Mechanical allodynia}

The von Frey method was performed weekly for 28 days in order to evaluate mechanical allodynia in the hind paws

Fig. 3 Line chart of von Frey filaments test scores of rats treated with metformin $(10,50$ and $100 \mathrm{mg} / \mathrm{kg})$ and minocycline. a Metformin at dose of $50 \mathrm{mg} / \mathrm{kg}$ significantly improved the resistance to mechanical pressures through 28 days after injury. There was no significant difference between metformin $(50 \mathrm{mg} / \mathrm{kg})$ and minocycline-treated animals in the study period. $\mathbf{b}$ The data are presented as mean \pm SD (6-8 rats per groups). $* P<0.05$, $* * P<0.01$, and $* * * * P<0.0001$ compared to the control group on the same day

after SCI (Fig. 3). The 50\% withdrawal threshold changes in the SHAM-operated animals were unremarkable through the study while the thresholds dropped significantly in SCIinduced animals a week after surgery (Mean difference: 33.55 , SD difference: $14.72, p<0.001)$. Weekly changes in mechanical sensitivity were assessed with repeated measure ANOVA. Our results revealed that, time $(p<0.001)$ and its interaction with treatment groups $(p<0.001)$ were significantly effective to improve resistance to mechanical pressure. Also, there was a significant difference between the effectiveness of different treatments $(F(5,34)=35.717$, 
$p<0.001)$. Metformin $50 \mathrm{mg} / \mathrm{kg}(p=0.016)$ and minocycline $(p<0.001)$ similarly improved the mechanical pain through time $(p=0.298)$, while none of them could restore the $50 \%$ threshold to the same level as SHAM group $(p<$ 0.001 ).

\section{Body weight}

After surgery, all SCI-induced animals suffered a significant weight loss of $12.4 \mathrm{~g}$ (S.E: 1.33 ) in 1 week. Moreover, repeated measure ANOVA reveled that through the 28 days, all of the rodents with SCI experienced a significant weight loss of $26.45 \mathrm{~g}$ (S.E: 3.56$)(p<0.001)$. Statistical analysis showed a significant weight gain in SHAM-operated animals (Mean difference: 32, S.E: 0.87, $p<0.001$ ). Repeated measure ANOVA for of all the studied rodents showed that weight loss was statistically similar in metformin-treated rats and the control group $(p>$ 0.05 ); however, weight loss in minocycline-treated rodents was significantly less than the control group (Mean difference: 14.51, S.E: $4.12, p=0.014)$. Comparing the effect of different interventions on weight changes, our results revealed that metformin at the dose of $50 \mathrm{mg} / \mathrm{kg}$ was more effective than the other applied doses of this drug $(p<0.01)$ (Fig. 4).

\section{Spinal histopathologic scores}

Cumulative histopathologic scores of $0-12$, based on hemorrhage followed by inflammatory cell invasion, neuronal vacuolation, and cyst formation were evaluated, by a blinded expert pathologist (Fig. 5). There was a statistically significant difference between groups as determined by oneway ANOVA $(F(2,23)=15.122, p<0.001)$. A Tukey post hoc test revealed that the histopathologic scores were significantly lower in metformin $50 \mathrm{mg} / \mathrm{kg}$ (Mean: 2.37, SD: 1.06, $p<0.001$ ) and minocycline (Mean: 3.75, SD: 1.48, $p=.006$ ) compared to the control group (Mean: 6.25, SD: 1.66). Additionally, there was no statistically significant difference between the metformin $50 \mathrm{mg} / \mathrm{kg}$ and minocycline groups $(p=0.157)$ (Table 1$)$.

\section{Cytokines assessment}

Proinflammatory cytokines, TNF- $\alpha$, and IL- $1 \beta$, were assessed in spinal cord 28 days after surgery to determine the potential anti-inflammatory effect of metformin in SCI rats. As expected, both TNF- $\alpha$, and IL- $1 \beta$ levels were significantly higher in control group (TNF- $\alpha$ : $631 \pm 16.39 \mathrm{pg} / \mathrm{mg}$, IL-1 1 : $374.26 \pm 36.30 \mathrm{pg} / \mathrm{mg}$ ) compared to metformin $50 \mathrm{mg} / \mathrm{kg}$ (TNF- $\alpha: \quad 8.09 \pm 1.15 \mathrm{pg} / \mathrm{mg}, \quad p<0.001, \quad \mathrm{IL}-1 \beta: \quad 95.13 \pm$ $36.30 \mathrm{pg} / \mathrm{mg}, \quad p<0.001)$ and minocycline (TNF- $\alpha: 4.99 \pm$ $1.54 \mathrm{pg} / \mathrm{mg}, \quad p<0.001, \quad \mathrm{IL}-1 \beta: 109.01 \pm 12.59 \mathrm{pg} / \mathrm{mg}, \quad p<$

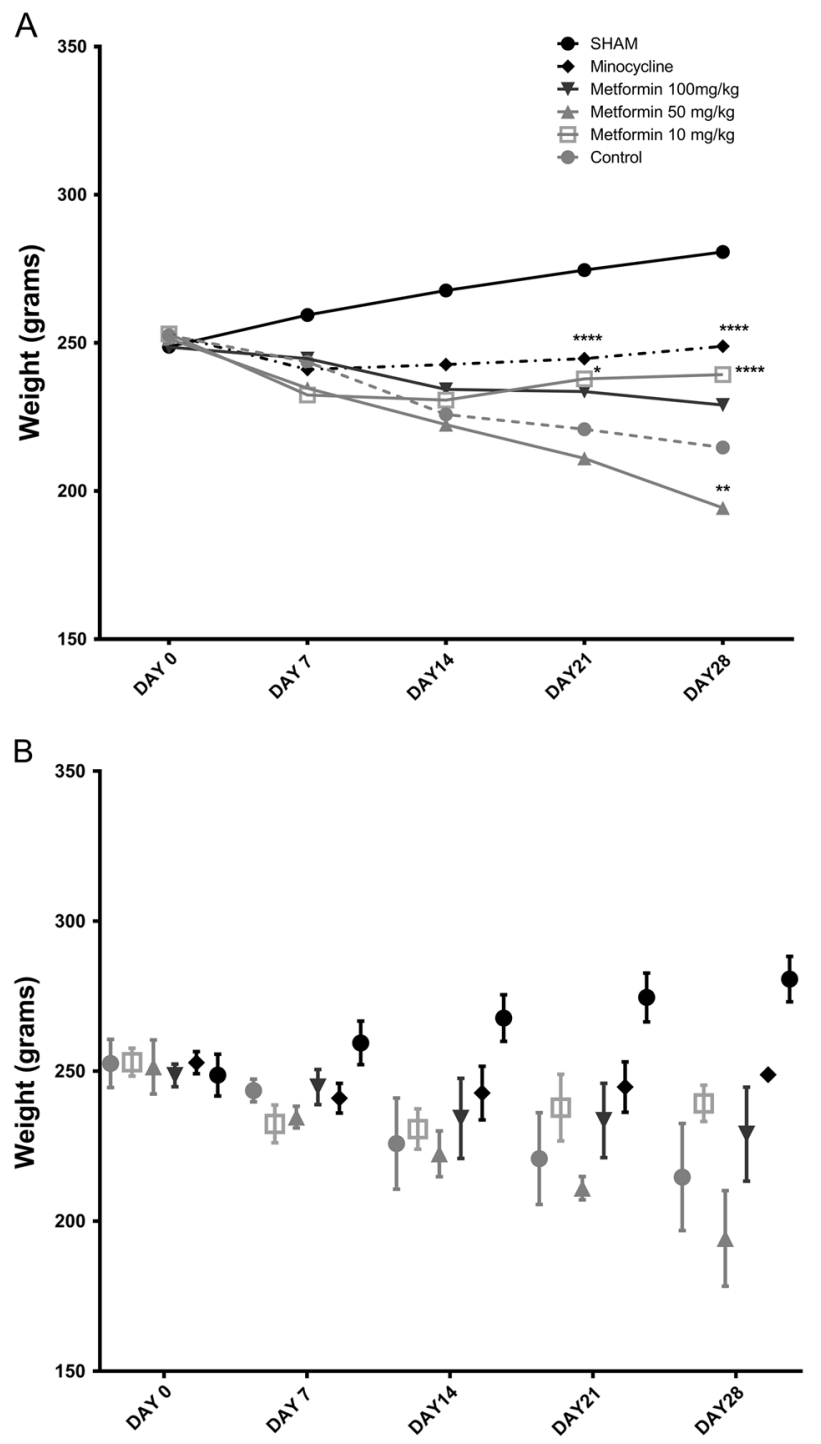

Fig. 4 Line chart of mean weight of rats received metformin (10, 50 and $100 \mathrm{mg} / \mathrm{kg}$ ) and minocycline. a Weight loss was similar in metformin $(50 \mathrm{mg} / \mathrm{kg})$-treated rats and the control group. Weight loss in minocycline group was significantly less than the control group. b The data are presented as mean $\pm \mathrm{SD}$ (6-8 rats per groups). $* P<0.05, * * P<0.01$, and $* * * * P<0.0001$ compared to the control group on the same day

Table 1 Cumulative spinal histopathological scores 28 days after injury in control, metformin and minocycline groups

\begin{tabular}{ll}
\hline Treatment Groups & Histopathologic scores \\
\hline control & $6.25 \pm 1.66$ \\
Metformin $(50 \mathrm{mg} / \mathrm{kg})$ & $2.37 \pm 1.06^{* * *}$ \\
Minocycline $(180 \mathrm{mg} / \mathrm{kg})$ & $3.75 \pm 1.48^{* *}$
\end{tabular}

$* *$ Significantly different from control $(p<0.01), * * *(p<0.001)$ 


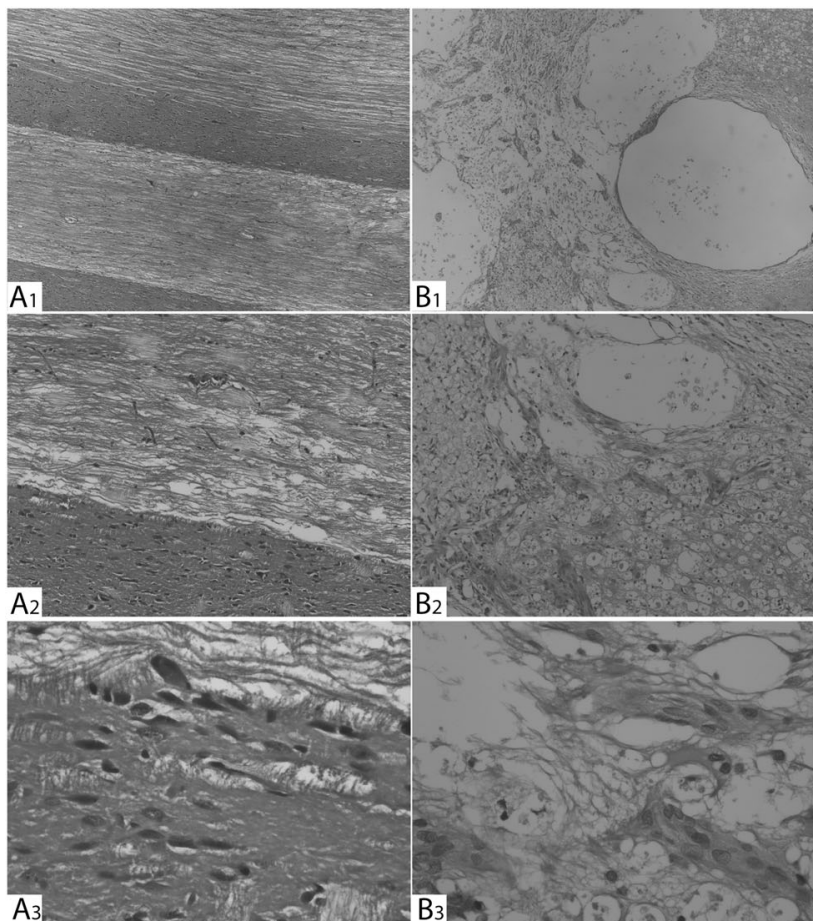

Fig. 5 Representative histopathology of spinal cord sections at the injury site 28 days after injury. Sections were examined by H\&E staining and the presence of inflammatory cells, hemorrhage, tissue degeneration and the extent of cyst formation were quantified. There are three rows of images with magnification of $\times 40, \times 100, \times 400$, respectively. a1-3 Represented SHAM group with normal

Table 2 Levels of inflammatory cytokines in rat's spine 28 days after injury in control, metformin and minocycline groups

\begin{tabular}{|c|c|c|c|}
\hline $\begin{array}{l}\text { Cytokine } \\
\text { (pg/mg) }\end{array}$ & Control & $\begin{array}{l}\text { Metformin } \\
(50 \mathrm{mg} / \mathrm{kg})\end{array}$ & $\begin{array}{l}\text { Minocycline } \\
(180 \mathrm{mg} / \mathrm{kg})\end{array}$ \\
\hline$N F-\alpha$ & $631 \pm 16.39$ & $8.09 \pm 1.15 * * *$ & $4.99 \pm 1.54 * * *$ \\
\hline IL-1 $\beta$ & $374.26 \pm 36.30$ & $95.13 \pm 36.30 * * *$ & $109.01 \pm 12.59 * * *$ \\
\hline
\end{tabular}

$T N F-\alpha$ Tumor necrosis factor alpha, $I L-1 \beta$ interleukin- $1 \beta$

$* * *$ Significantly different from control $(p<0.001)$

0.001).Moreover, there was no significant difference in cytokine levels between metformin $50 \mathrm{mg} / \mathrm{kg}$ and minocycline administration $(p \quad$ TNF- $\alpha=0.894, \quad p \quad$ IL-1 $1 \beta=0.781)$ (Table 2).

\section{Correlation of locomotor score and pain assessments}

Our obtained data indicate that the increase of locomotion (BBB score) is significantly correlated with a decrease in sensory response in von Frey filament test in spinal cordinjured rodents (spearman correlation coefficient $=-0.454$, $p$ value $=0.01$, which is also significantly correlated with a

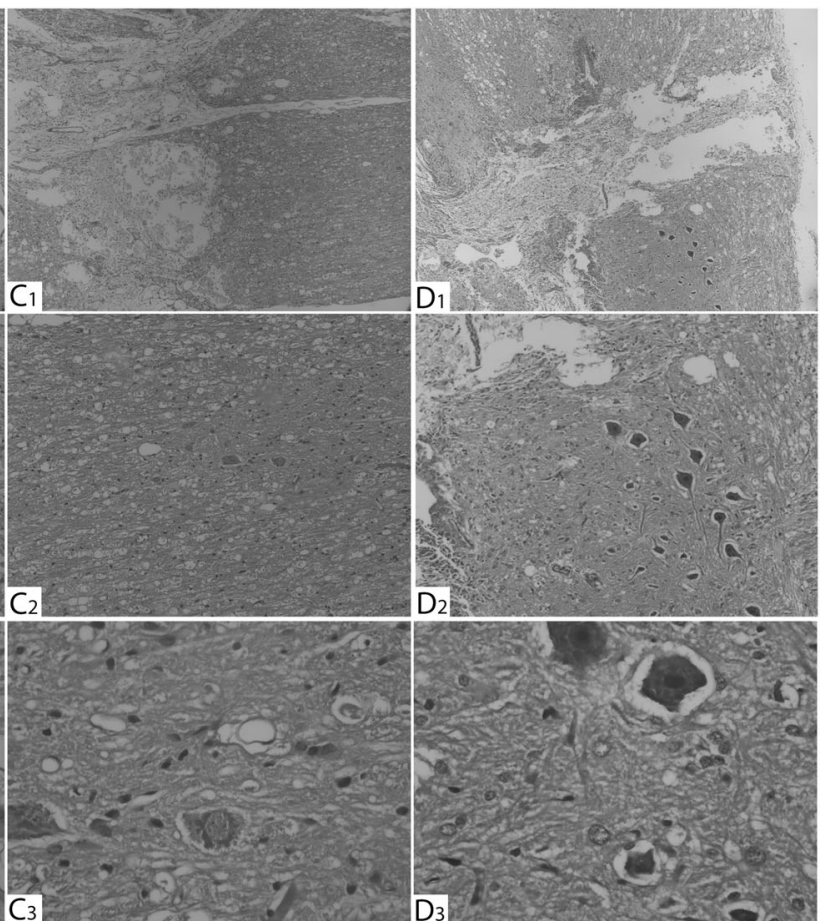

histopathologic assessment. Control group (b1-3) shows significant cyst formation, immune cell infiltration and vacuolization in the cord. Minocycline group (c1-3) elucidating significant vacuolization and minor cyst formation. However Metformin (50 mg/kg) group (d1-3), showing minor vacuolization and immune cell infiltration

decrease in tail flick latency (spearman correlation coefficient $=0.465, p$ value $=0.02$ )

\section{Discussion}

The current study demonstrated that metformin, administrated at a single dose of $50 \mathrm{mg} / \mathrm{kg}$ at the time of injury, had significant effects on SCI complications. Our results showed that in addition to significant improvement in behavioral responses, metformin substantially decreased histopathological signs of neuroinflammation and the level of TNF- $\alpha$ and IL-1 $\beta$ inflammatory cytokines in the cord tissue of spinal cord-injured rats. Interestingly, the therapeutic effects of metformin were similar to minocycline, a potential neuroprotective agent for SCI [5, 13].

In the current study, tactile and heat stimulation tests were started a week after surgery when according to previous studies acute inflammation is reviled and regeneration is initiated [17]. Comparing our results on the progress of locomotor improvement with previous studies, it could be concluded that the severity of SCI could not have affected the animals' response to neuropathic pain [18, 19]. Interestingly, our results showed that while all of the animals' 
locomotor activity was improved through time, metformin $50 \mathrm{mg} / \mathrm{kg}$ and minocycline lead to a significant increase in TFL and VF response in contrast to the control group. Thus, these findings confirm that the applied treatments improved the pain threshold contrary to the expected course in SCI injured rodents.

SCI leads to acute and chronic neuroinflammation associated with neurotoxicity, axonal damage and finally glial scarring. The scale of inflammation is directly associated with the release of inflammatory cytokines and also the amount of hemorrhage caused by SCI [20, 21]. It is believed that cyst formation and glial scar, the most important inhibitors for neuroregeneration, are mostly formed by reactive astrocytes, macrophages and microglia [21]. The gradual increased inflammatory activation of these cells peaking 4 weeks after injury aggravates neuronal death and axonal damage. Inflammatory cytokines such as TNF- $\alpha$ and IL-1 $\beta$ are proposed to have a major role in this process [20, 21]. Moreover, the post-SCI neuronal-glial interactions associated with maladaptive synaptic plasticity and cellular signaling and hyperactivity of sensory neurons contribute to the heightening of the neuropathic pain after SCI [22]. The histopathological evaluations in the current study showed that metformin and minocycline had similar effects on the spinal cord tissue, as they both significantly reduced inflammation, vacuolation, hemorrhage and cyst formation compared to the control group. The similar improvement of the functional behavior and reduced responsiveness to mechanical and thermal allodynia also verifies the hypothesized therapeutic effects of these treatments. Also, the similarity of our findings with previous studies on the efficacy of metformin and minocycline in SCI functional recovery could confirm the validity of these interventions[7, 23, 24]. These results are consistent with previous research showing that inhibition of proinflammatory cytokines has significant protective effects on neuronal death and axonal damage and improves healing in the regenerative process [21].

Studies have shown that microglial inhibition via neutralization of TNF- $\alpha$ and IL- $1 \beta$ attenuates neuropathic pain and mechanical hyperalgesia. Minocycline, described to be therapeutic in various pain facilitation studies, is considered to be an effective agent through inhibition of these cytokines, and thus reduced microglial activity [25]. Similar to the current research, investigations indicate that metformin inhibits these inflammatory cytokines (e.g., TNF- $\alpha$ ) and their receptors; specifically in immune cells such as macrophages [26]. Metformin has neuroprotective effects leading to both functional recovery and neural salvage in different CNS impairments such as stroke, Parkinson's disease, and SCI [7, 27]. In addition, regulation of autophagy by metformin promotes neuronal survival and functional recovery in spinal cord injury [7]. Likewise, minocycline inhibits inflammatory cytokines such as TNF$\alpha$. The attenuation of inflammatory cytokines by minocycline in addition to the anti-apoptotic properties of this agent are shown to have significant effect in SCI complications $[13,28,29]$. In the present study, the similar curative influence of metformin and minocycline in SCI-induced hyperalgesia, considering the akin significantly diminished histopathological impairment and proinflammatory TNF- $\alpha$ and IL- $1 \beta$ cytokines levels, promotes the hypothesis of an analogous protective interaction between these drugs. Recent researches have indicated that the normal inflammatory-regenerative sequence of tissue repair process is impaired in SCI [30]. It has been described that high levels of TNF- $\alpha$ lead to neurotoxicity, axonotomy and promotes inflammation [31]. The positive loop of cytokines generation leads to a continuous neurotoxicity and chronic inflammation, cessation of this vicious cycle, as shown by the results of the current study, would be beneficial for slowing and reversing the course of the impairment [30, 32].

Multiple signaling pathways regulate the NF- $\mathrm{KB}$ levels, which have a major role in SCI inflammation and neurotoxicity, in cells affected by cord injury [33, 34]. Some of these signaling pathways are the mitogen-activated protein kinase (MAPK) p38, the phosphoinositide-3-kinase (PI3K) and mTOR $[35,36]$. It has been demonstrated that metformin as an inhibitor of mTOR attenuates neuronal damage and locomotor impairment, regulates autophagy and inhibits the NF-kappaB signaling [7, 8]. Besides blocking the inflammatory cytokines such as TNF- $\alpha$, minocycline has been reported to play its protective role by regulation of $\mathrm{P} 38$ MAPK and (PI3K)/Akt together with NF-kappaB signaling pathways in the spinal cord [28, 29, 37]. Taken together, it could be suggested that inhibition of NF-kappaB signaling is a possible common pathway for the protective effect of metformin and minocycline. Although, as there are many cellular and pathological pathways in spinal cord injury, the exact evaluation of the protective mechanisms involved in treatment by these drugs were out of the scopes of the current research $[38,39]$. However, regarding the discussed similarities resulted from previous research and the current study, it could be concluded that metformin and minocycline attenuate the pathological and behavior outcomes of spinal cord injury through similar pathways with antiinflammatory characteristics being prominent among them. However, the differences between these therapeutic approaches would be a noteworthy query.

Significant weight loss is an acute characteristic of spinal cord injury, which occurs due to the metabolic changes, nutritional markers depletion and muscle atrophy [40]. Following the acute weight loss subjects suffering from immobility come into a weight gaining phase, which is mostly promoted by increased body fat and visceral 
adiposity that may lead to difficult challenges such as metabolic syndrome and cardiovascular complications [41]. In this study, minocycline significantly reversed the weight loss compared to control group. On the other hand, metformin administration was associated with significant weight loss. The contrast between the effects of these therapies could be implied by the fact that metformin is known to cause weight loss by lowering food intake through different pharmacological interactions such as improving insulin sensitivity and also improving lipid metabolism [42]. Considering the negative effects of weight gain in SCI, the weight losing characteristic of this drug, thus might be beneficial after cord injury. Indeed, more extensive studies with a focus on weight changes would be imperative for confirmation of these effects.

It has been demonstrated that administration of metfor$\mathrm{min}$ at the dose of $50 \mathrm{mg} / \mathrm{kg}$ shows a more prominent efficacy in attenuating IL-1 $\beta$ and TNF-alpha levels, compared to high doses of 100 and $200 \mathrm{mg} / \mathrm{kg}$. Also, by correlating the drug dosage from animal to human dose, the antiinflammatory dose of $50 \mathrm{mg} / \mathrm{kg}$ metformin in rodents is shown to be below the therapeutic dose used in diabetic humans [43]. Interestingly, study of metformin's doses response curve has shown to have a biphasic effect in the efficacy of the drug. According to this curve, the efficacy of metformin decreases at both very low and high doses [44]. Our results, in accordance with the mentioned previous findings, indicate that metformin at the dose of $50 \mathrm{mg} / \mathrm{kg}$ had the highest effect on SCI complications compared to other administrated doses in this study.

In summary, we showed that metformin significantly improves locomotor activity and neuropathic pain in spinal cord-injured rodents through the attenuation of destructive neuroinflammatory responses. According to our results, metformin might be beneficial in post-SCI weight changes. We also discussed similarities in therapeutic effects of metformin and minocycline following SCI. The similarities include improving the functional recovery, histopathological and inflammatory status of the injured cord and neuropathic pain. Moreover, considering the similar therapeutic effects of metformin and minocycline in addition to its presumably favorable post-SCI weight changes, metformin's smaller production price, wider therapeutic window and lower incidence of side effects, the authors strongly suggest further investigations of the potential clinical therapeutic effects of metformin on spinal cord injury in human.

Acknowledgements This study was financially supported by Experimental Medicine Research Center, Tehran University of Medical Sciences, Tehran, Iran (Grant No 95-01-30-31469) and by a grant (96002757) from the Iran National Science Foundation (INSF). We gratefully acknowledge the dedicated efforts of the investigators including Dr. Mohsen Afarideh and the coordinators who participated in this study.

\section{Compliance with ethical standards}

Conflict of interest The authors declare that they have no conflict of interest.

\section{References}

1. Anwar MA, Al Shehabi TS, Eid AH. Inflammogenesis of secondary spinal cord injury. Front Cell Neurosci. 2016;10:98.

2. Faden AI, Wu J, Stoica BA, Loane DJ. Progressive inflammationmediated neurodegeneration after traumatic brain or spinal cord injury. Br J Pharmacol. 2016;173:681-91.

3. Nees TA, Finnerup NB, Blesch A, Weidner N. Neuropathic pain after spinal cord injury: the impact of sensorimotor activity. Pain. 2017;158:371-6.

4. Tateda S, Kanno H, Ozawa H, Sekiguchi A, Yahata K, Yamaya S, et al. Rapamycin suppresses microglial activation and reduces the development of neuropathic pain after spinal cord injury. J Orthop Res. 2017;35:93-103.

5. Marchand F, Tsantoulas C, Singh D, Grist J, Clark AK, Bradbury EJ, et al. Effects of Etanercept and Minocycline in a rat model of spinal cord injury. Eur J Pain. 2009;13:673-81.

6. Howell JJ, Hellberg K, Turner M, Talbott G, Kolar MJ, Ross DS, et al. Metformin inhibits hepatic mTORC1 signaling via dosedependent mechanisms involving AMPK and the TSC complex. Cell Metab. 2017;25:463-71.

7. Zhang D, Xuan J, Zheng B-b, Zhou Y-1, Lin Y, Wu Y-s, et al. Metformin improves functional recovery after spinal cord injury via autophagy flux stimulation. Mol Neurobiol. 2017;54: $3327-41$.

8. Wang C, Liu C, Gao K, Zhao H, Zhou Z, Shen Z, et al. Metformin preconditioning provide neuroprotection through enhancement of autophagy and suppression of inflammation and apoptosis after spinal cord injury. Biochem Biophys Res Commun. 2016;477:534-40.

9. Pan Y, Sun X, Jiang L, Hu L, Kong H, Han Y, et al. Metformin reduces morphine tolerance by inhibiting microglial-mediated neuroinflammation. J Neuroinflamm. 2016;13:294.

10. Łabuzek K, Gabryel B, Okopień B. Metformin as a key to alternative activation of microglia? Post Hig Med Dosw (Online). 2014;68:247-57.

11. Oliveira WH, Nunes AK, Franca ME, Santos LA, Los DB, Rocha $\mathrm{SW}$, et al. Effects of metformin on inflammation and short-term memory in streptozotocin-induced diabetic mice. Brain Res. 2016;1644:149-60.

12. Inyang K,Szabo-Pardi T,Price $T$, Treatment of Chronic pain: long term effects of Metformin on chronic neuropathic pain and microglial activation. J Pain. 2016;17:S53

13. Lee SM, Yune TY, Kim SJ, Park DW, Lee YK, Kim YC, et al. Minocycline reduces cell death and improves functional recovery after traumatic spinal cord injury in the rat. $\mathrm{J}$ Neurotrauma. 2003;20:1017-27.

14. Basso DM, Beattie MS, Bresnahan JC. A sensitive and reliable locomotor rating scale for open field testing in rats. J Neurotrauma. 1995;12:1-21.

15. Qiao F, Atkinson C, Kindy MS, Shunmugavel A, Morgan BP, Song $\mathrm{H}$, et al. The alternative and terminal pathways of complement mediate post-traumatic spinal cord inflammation and injury. Am J Pathol. 2010;177:3061-70.

16. Gonzalez R, Glaser J, Liu MT, Lane TE, Keirstead HS. Reducing inflammation decreases secondary degeneration and functional deficit after spinal cord injury. Exp Neurol. 2003;184:456-63.

17. Kanno H, Ozawa H, Sekiguchi A, Yamaya S, Tateda S, Yahata K, et al. The role of mTOR signaling pathway in spinal cord injury. Cell Cycle. 2012;11:3175-9. 
18. Lee-Kubli CA, Ingves M, Henry KW, Shiao R, Collyer E, Tuszynski $\mathrm{MH}$, et al. Analysis of the behavioral, cellular and molecular characteristics of pain in severe rodent spinal cord injury. Exp Neurol. 2016;278:91-104.

19. Shinozaki M, Iwanami A, Fujiyoshi K, Tashiro S, Kitamura K, Shibata $\mathrm{S}$, et al. Combined treatment with chondroitinase ABC and treadmill rehabilitation for chronic severe spinal cord injury in adult rats. Neurosci Res. 2016;113:37-47.

20. Rooney GE, Endo T, Ameenuddin S, Chen B, Vaishya S, Gross L, et al. Importance of the vasculature in cyst formation after spinal cord injury. J Neurosurg: Spine. 2009;11: 432-7.

21. Yuan Y-M, He C. The glial scar in spinal cord injury and repair. Neurosci Bull. 2013;29:421-35.

22. Gwak YS, Hulsebosch CE, Leem JW. Neuronal-Glial interactions maintain chronic neuropathic pain after spinal cord injury. Neural Plast. 2017; 2017:2480689. https://www.hindawi.com/journals/ np/2017/2480689/

23. Festoff BW, Ameenuddin S, Arnold PM, Wong A, Santacruz KS, Citron BA. Minocycline neuroprotects, reduces microgliosis, and inhibits caspase protease expression early after spinal cord injury. J Neurochem. 2006;97:1314-26.

24. Yune TY, Lee JY, Jung GY, Kim SJ, Jiang MH, Kim YC, et al. Minocycline alleviates death of oligodendrocytes by inhibiting pro-nerve growth factor production in microglia after spinal cord injury. J Neurosci. 2007;27:7751-61.

25. Bradesi S. Role of spinal cord glia in the central processing of peripheral pain perception. Neurogastroenterol \& Motil. 2010;22:499-511.

26. Hyun B, Shin S, Lee A, Lee S, Song Y, Ha NJ, et al. Metformin Down-regulates TNF-alpha Secretion via Suppression of Scavenger Receptors in Macrophages. Immune Netw. 2013;13:123-32.

27. Song GJ, Suk K. Pharmacological modulation of functional phenotypes of microglia in neurodegenerative diseases. Front Aging Neurosci. 2017;9:139.

28. Gong K, Zou X, Fuchs PN, Lin Q. Minocycline inhibits neurogenic inflammation by blocking the effects of tumor necrosis factor- $\alpha$. Clin Exp Pharmacol Physiol. 2015;42:940-9.

29. Shultz RB, Zhong Y. Minocycline targets multiple secondary injury mechanisms in traumatic spinal cord injury. Neural Regen Res. 2017;12:702.

30. Gensel JC, Zhang B. Macrophage activation and its role in repair and pathology after spinal cord injury. Brain Res. 2015; 1619:1-11.
31. Kuno R, Wang J, Kawanokuchi J, Takeuchi H, Mizuno T, Suzumura A. Autocrine activation of microglia by tumor necrosis factor-alpha. J Neuroimmunol. 2005;162:89-96.

32. Pugazhenthi S, Zhang Y, Bouchard R, Mahaffey G. Induction of an inflammatory loop by interleukin- $1 \beta$ and tumor necrosis factor$\alpha$ involves NF-kB and STAT-1 in differentiated human neuroprogenitor cells. PLoS ONE. 2013;8:e69585.

33. Engelmann C, Weih F, Haenold R. Role of nuclear factor kappa B in central nervous system regeneration. Neural Regen Res. 2014;9:707.

34. Bracchi-Ricard V, Lambertsen KL, Ricard J, Nathanson L, Karmally S, Johnstone J, et al. Inhibition of astroglial NF-kappaB enhances oligodendrogenesis following spinal cord injury. J Neuroinflamm. 2013;10:869.

35. Schulze-Osthoff K, Ferrari D, Riehemann K, Wesselborg S. Regulation of NF-kappa B activation by MAP kinase cascades. Immunobiology. 1997;198:35-49.

36. Ahmad A, Biersack B, Li Y, Kong D, Bao B, Schobert R, et al. Targeted regulation of $\mathrm{PI} 3 \mathrm{~K} / \mathrm{Akt} / \mathrm{mTOR} / \mathrm{NF}-\kappa \mathrm{B}$ signaling by indole compounds and their derivatives: mechanistic details and biological implications for cancer therapy. Anticancer Agents Med Chem. 2013;13:1002-13.

37. Song Z-p, Xiong B-r, Guan X-h, Cao F, Manyande A, Zhou Y-q, et al. Minocycline attenuates bone cancer pain in rats by inhibiting NF-KB in spinal astrocytes. Acta Pharmacol Sin. 2016;37:753-62.

38. Zhang $\mathrm{H}$, Wang $\mathrm{Y}$. Identification of molecular pathway changes after spinal cord injury by microarray analysis. J Orthop Surg. 2016;11:101.

39. Wu J, Stoica BA, Faden AI. Cell cycle activation and spinal cord injury. Neurotherapeutics. 2011;8:221-8.

40. Thibault-Halman G, Casha S, Singer S, Christie S. Acute management of nutritional demands after spinal cord injury. J Neurotrauma. 2011;28:1497-507.

41. Powell D, Affuso O, Chen Y. Weight change after spinal cord injury. J Spinal Cord Med. 2017;40:130-7.

42. Malin SK, Kashyap SR. Effects of metformin on weight loss: potential mechanisms. Curr Opin Endocrinol Diabetes Obes. 2014;21:323-9.

43. de Araújo AA, Pereira AdSBF, de Medeiros CACX, de Castro Brito GA, de Carvalho Leitão RF, de Souza Araújo L, et al. Effects of metformin on inflammation, oxidative stress, and bone loss in a rat model of periodontitis. PLoS ONE. 2017;12:e0183506.

44. Riddle M, Combining sulfonylureas and other oral agents. Am J Med. 2000;108:15s-22. 